University of Wollongong

Research Online

Australian Institute for Innovative Materials -

Papers

Australian Institute for Innovative Materials

$1-1-2013$

Organic solvent-based graphene oxide liquid crystals: A facile route toward the next generation of self-assembled layer-by-layer multifunctional 3D architectures

\author{
Rouhollah Jalili \\ University of Wollongong, rjalili@uow.edu.au \\ Seyed Hamed Aboutalebi \\ University of Wollongong, sha942@uowmail.edu.au \\ Dorna Esrafilzadeh \\ University of Wollongong, de256@uowmail.edu.au \\ Konstantin Konstantinov \\ University of Wollongong, konstan@uow.edu.au \\ Simon E. Moulton \\ University of Wollongong, smoulton@uow.edu.au
}

See next page for additional authors

Follow this and additional works at: https://ro.uow.edu.au/aiimpapers

Part of the Engineering Commons, and the Physical Sciences and Mathematics Commons

Research Online is the open access institutional repository for the University of Wollongong. For further information contact the UOW Library: research-pubs@uow.edu.au 


\title{
Organic solvent-based graphene oxide liquid crystals: A facile route toward the next generation of self-assembled layer-by-layer multifunctional 3D architectures
}

\begin{abstract}
We introduce soft self-assembly of ultralarge liquid crystalline (LC) graphene oxide (GO) sheets in a wide range of organic solvents overcoming the practical limitations imposed on LC GO processing in water. This expands the number of known solvents which can support amphiphilic self-assembly to ethanol, acetone, tetrahydrofuran, $\mathrm{N}$-dimethylformamide, $\mathrm{N}$-cyclohexyl-2-pyrrolidone, and a number of other organic solvents, many of which were not known to afford solvophobic self-assembly prior to this report. The LC behavior of the as-prepared GO sheets in organic solvents has enabled us to disperse and organize substantial amounts of aggregate-free single-walled carbon nanotubes (SWNTs, up to 10 wt \%) without compromise in LC properties. The as-prepared LC GO-SWNT dispersions were employed to achieve self-assembled layer-by-layer multifunctional 3D hybrid architectures comprising SWNTs and GO with unrivalled superior mechanical properties (Young's modulus in excess of $50 \mathrm{GPa}$ and tensile strength of more than $500 \mathrm{MPa}$ ). 2013 American Chemical Society.
\end{abstract}

\section{Keywords}

generation, self, assembled, crystals, organic, solvent, facile, graphene, oxide, layer, liquid, multifunctional, architectures, $3 d$, route, toward, next

Disciplines

Engineering | Physical Sciences and Mathematics

\section{Publication Details}

Jalili, R., Aboutalebi, S., Esrafilzadeh, D., Konstantinov, K. K., Moulton, S. E., Razal, J. M. \& Wallace, G. G. (2013). Organic solvent-based graphene oxide liquid crystals: A facile route toward the next generation of self-assembled layer-by-layer multifunctional 3D architectures. ACS Nano, 7 (5), 3981-3990.

\section{Authors}

Rouhollah Jalili, Seyed Hamed Aboutalebi, Dorna Esrafilzadeh, Konstantin Konstantinov, Simon E. Moulton, Joselito M. Razal, and Gordon G. Wallace 


\section{Organic Solvent-Based Graphene Oxide Liquid}

\section{Crystals: A Facile Route Towards Next Generation}

\section{of Self-Assembled Layer-by-Layer Multifunctional}

\section{D Architectures}

By Rouhollah Jalili ${ }^{\ddagger 1}$, Seyed Hamed Aboutalebi ${ }^{\ddagger 2}$, Dorna Esrafilzadeh ${ }^{1}$, Konstantin

Konstantinov ${ }^{2}$, Simon E. Moulton ${ }^{1}$, Joselito M. Razal ${ }^{1}$ and Gordon G. Wallace ${ }^{1 *}$

1, Intelligent Polymer Research Institute, ARC Centre of Excellence for Electromaterials

Science, AIIM Facility, Innovation Campus, University of Wollongong, North Wollongong, NSW 2522 (Australia)

2, Institute for Superconducting and Electronic Materials, AIIM Facility, Innovation Campus, University of Wollongong, North Wollongong, NSW 2522 (Australia)

*To whom correspondence should be addressed. Email: gwallace@uow.edu.au

ABSTRACT. We introduce soft self-assembly of ultra-large liquid crystalline (LC) graphene oxide (GO) sheets in a wide range of organic solvents overcoming the practical limitations imposed on LC GO processing in water. This expands the number of known solvents which can support amphiphilic self-assembly to ethanol, acetone, tetrahydrofuran, N-dimethylformamide, 
$\mathrm{N}$-cyclohexyl-2-pyrrolidone and a number of other organic solvents, many of which were not known to afford solvophobic self-assembly prior to this report. The LC behavior of the asprepared GO sheets in organic solvents has enabled us to disperse and organize substantial amounts of aggregate-free single walled carbon nanotubes (SWNTs, up to $10 \mathrm{wt}$. \%) without compromise in LC properties. The as-prepared LC GO-SWNTs dispersions were employed to achieve self-assembled layer-by-layer multifunctional 3D hybrid architectures comprising of SWNTs and GO with unrivalled superior mechanical properties (Young's modulus in excess of 50 GPa and tensile strength of more than $500 \mathrm{MPa}$ ).

KEYWORDS: Graphene Oxide, Liquid crystal, Liquid crystal in organic solvent, Self-assembled composite, Layer-by-layer composite.

Lyotropic liquid crystalline (LC) phases in dispersions containing two dimensional graphene and graphene oxide (GO) sheets have added a new dimension to soft self-assembly science. ${ }^{1-9}$ Soft self-assembly of materials, which is the route for engineering of amphiphilic molecules into different supermolecular assemblies in one, two, or three dimensions, has been of interest for decades. ${ }^{10-12}$ Fields such as nano-medicine, biocatalysis, bio-active delivery systems, selfassembled composite and solar cells have taken advantage of the self-organization of amphiphilic molecules in recent years. ${ }^{13-16}$ Recently, a series of graphene based macroscopic structures including paper and fibers have been fabricated employing the novel amphiphilic soft self-assembly route. ${ }^{1,4,17}$ The much expected enhancement in properties, self-assembly and alignment of GO might be achieved if the simultaneous dispersion of functional materials with 
GO in the liquid crystalline media is realized. ${ }^{18}$ To date, the application of graphene-based liquid crystals as a promising building block in different fields has not yet been realized mainly because of the practical limitations induced by water, the only medium in which the formation of LC GO has been reported. However, there are limitations for aqueous media to disperse many nanomaterials that would be expected to introduce enhanced properties to LC GO. For example, the introduction of highly debundled, isolated and self-oriented carbon nanotubes (CNTs) which has only been observed in CHP and never in water with LC GO would be expected to enhance electrochemical and mechanical properties. Understanding and manipulating the forces involved in amphiphilic self-assembly and expanding the range of solvents in which such phenomena can be exploited, will enable the development of new composites based on LC GO. ${ }^{10}$

Here we report the ability to support GO lyotropic LC phase formation in a wide range of organic solvents through the use of ultra-large GO sheets. This approach enables the exploitation of LC order of GO sheets in organic solvents to organize and align single-walled carbon nanotubes (SWNTs). This work has enabled self-assembly of ultra-stiff, ultra-strong three dimensional (3D) GO-SWNT architectures with high elongation-at-break.

\section{Results and discussion}

\section{Formation of LC GO in various organic solvents}

To date, water is recognized as the practical medium for the self-assembly of GO. ${ }^{19}$ In the pursuit of rationally designed lyotropic LC GO dispersions in various organic solvents, we dispersed ultra-large GO sheets in a number of common solvents including: water, N-Ndimethylformamide (DMF), N-Cyclohexyl-2-pyrrolidone (CHP), Tetrahydrofuran (THF), acetone, ethanol and a number of other organic solvents, many of which were not known to afford solvophobic self-assembly prior to this report. The organic solvents, successfully used 
here, can support dissolution or dispersion of a wide range of materials. This means that selfassembly of a variety of compositions in the solvent media that support LC GO formation would be possible. For example, DMF and THF are the most common solvents for processing polymers. Therefore, dispersing GO in these solvents and achieving LC GO would provide unique opportunities in the production of self-assembled, fully ordered and novel LC GO-based polymer composites. LC GO in THF might also be used as an ordered template for the synthesis and self-assembly of metallic nano-particles such as boron or magnesium, which are water and air sensitive. LC GO in CHP, being the best known solvent for debundling CNTs, ${ }^{20}$ might promote fabrication of fully ordered self-assembled CNT-GO composites containing highly debundled CNTs. Ethanol and acetone are general purpose solvents which are commonly used as building blocks in organic chemistry. Acetone is also the solvent of choice for a wide range of epoxy families. Therefore, attaining LC GO in acetone would open an avenue for novel selfassembled epoxy based nano-composites. Other solvents in which we observed lyotropic LC formation of GO include ethylene glycol, methanol, acetonitrile, isopropanol, N-methyl pyrrolidone (NMP) and dimethyl acetamide (DMAc). All of the non-polar solvents were ineffective in dispersing GO and therefore could not afford any LC phase as expected. Representative polarized optical microscopy (POM) micrographs of the representative solvents are given in Figure 1. POM micrographs clearly show the birefringent lyotropic LC behavior of GO in water and all of the above-mentioned organic solvents (see also Figure S1 to S6). The transition concentration from isotropic to the nematic phase was experimentally found to be $\sim 0.25 \mathrm{mg} \mathrm{ml}^{-1}$ for water, DMF, CHP and ethanol, and $\sim 0.50 \mathrm{mg} \mathrm{ml}^{-1}$ for acetone and THF (Table 1). At higher concentrations, the nematic phase formed in all of the solvents spontaneously. Depending on the concentration, as-prepared organic LC GO can be either stable for months or 
undergo what is stated as degradation. ${ }^{21}$ It should be noted that these concentrations, although the lowest filler content ever reported for the formation of liquid crystals from any colloid, are still higher than the theoretical biphasic region between 0.05 to $0.09 \mathrm{mg} \mathrm{ml}^{-1}$ calculated for rigid platelets (see supporting information for details). This discrepancy can be attributed to the flexible nature of the monolayer GO sheets and their tendency of wrinkling especially in the presence of attached functional groups.
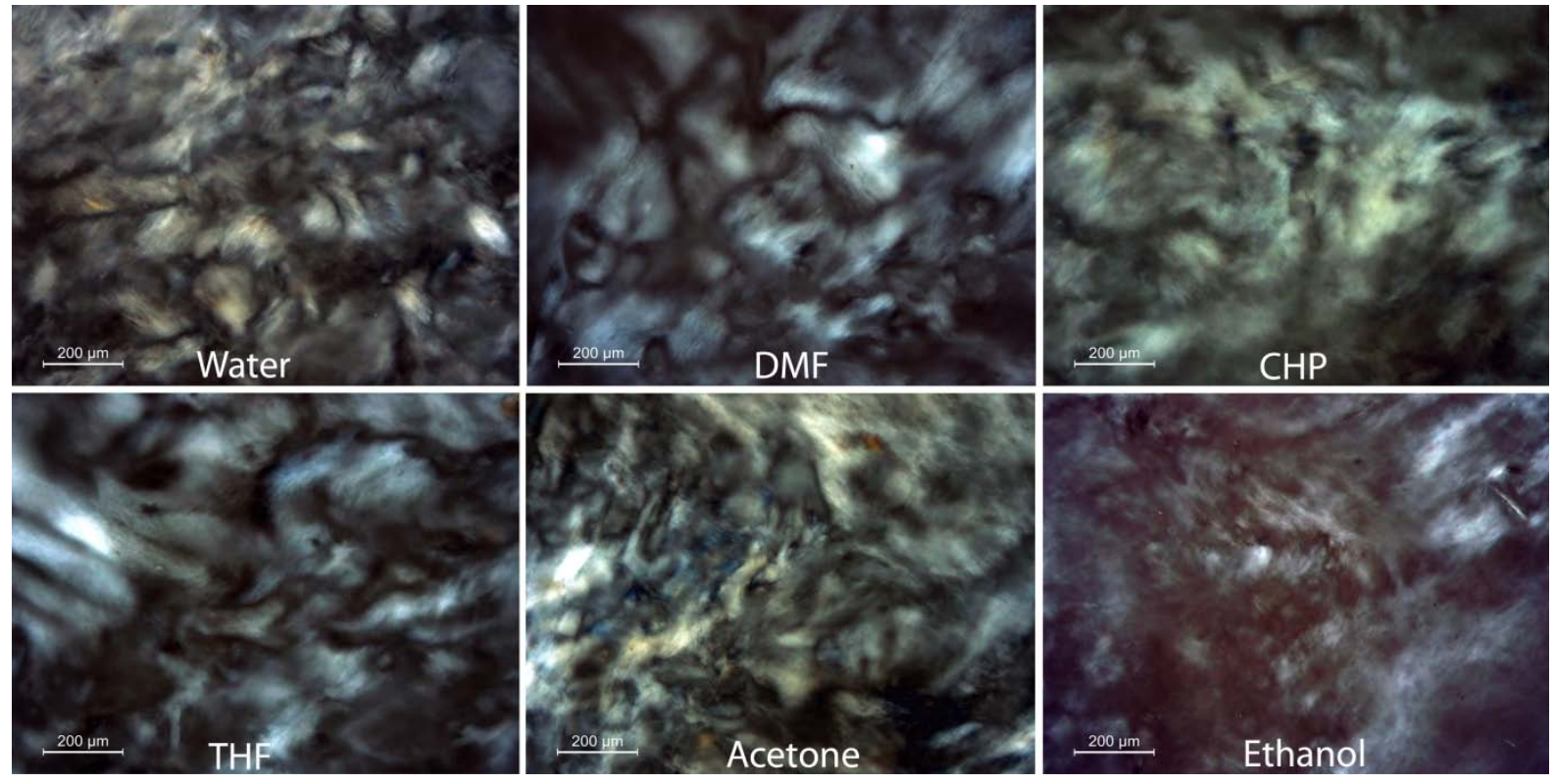

Figure 1. Representative crossed polarized optical microscopy (POM) of LC GO in various organic solvents at a GO concentration of $2.5 \mathrm{mg} \mathrm{ml}^{-1}$. 
Table 1. LC formation concentration and GO sheet properties in different solvents.

\begin{tabular}{llll}
\hline Solvent & LC formation concentration & $\begin{array}{l}\text { Sheet } \\
\text { thickness* }\end{array}$ & d-spacing** \\
\hline Water & $(\mathrm{mg}$ ml-1) & $(\mathrm{nm})$ & $(\mathrm{nm})$ \\
\cline { 2 - 4 } DMF & 0.25 & 0.83 & 0.825 \\
CHP & 0.25 & 1.1 & 1.05 \\
THF & 0.25 & 1.0 & 0.101 \\
Acetone & 0.50 & 0.92 & 0.937 \\
Ethanol & 0.50 & 0.86 & 0.846 \\
\hline
\end{tabular}

*: Sheet thickness obtained from AFM images

**:d-spacing obtained from XRD patterns

\section{Characterization of LC GO}

Atomic force microscopy was employed to assess the number of layers and quality of GO sheets in the organic solvent-based LC dispersions, (Figure 2). No aggregation or restacking of GO sheets was observed in any solvent investigated here. As-prepared GO dispersions in all of the organic solvents contained GO sheets that are predominantly in the size of more than tens of micrometres. Step height measurements performed on the samples indicated that all of the samples contained single layer GO sheets; as the mean measured height was between 0.8 to 1.2 $\mathrm{nm}$ depending on the solvent used. The thickness of a monolayer of graphene is about $0.34 \mathrm{~nm}$, 22 however, GO has functional groups that act as pillars giving rise to the larger measured height. Some solvent molecules are also expected to bond with the surface of GO and remain even after drying. Hydrogen bonding of each solvent molecule with GO resulted in different apparent sheet thickness, depending on the solvent molecular size and arrangement on the surface of GO sheets. 
Moreover, the crumpling of GO sheets that occurs in the case of THF (Figure 2) might also result in lower effective aspect ratio which manifests itself in the observation of different phase boundaries presented in Table 1.
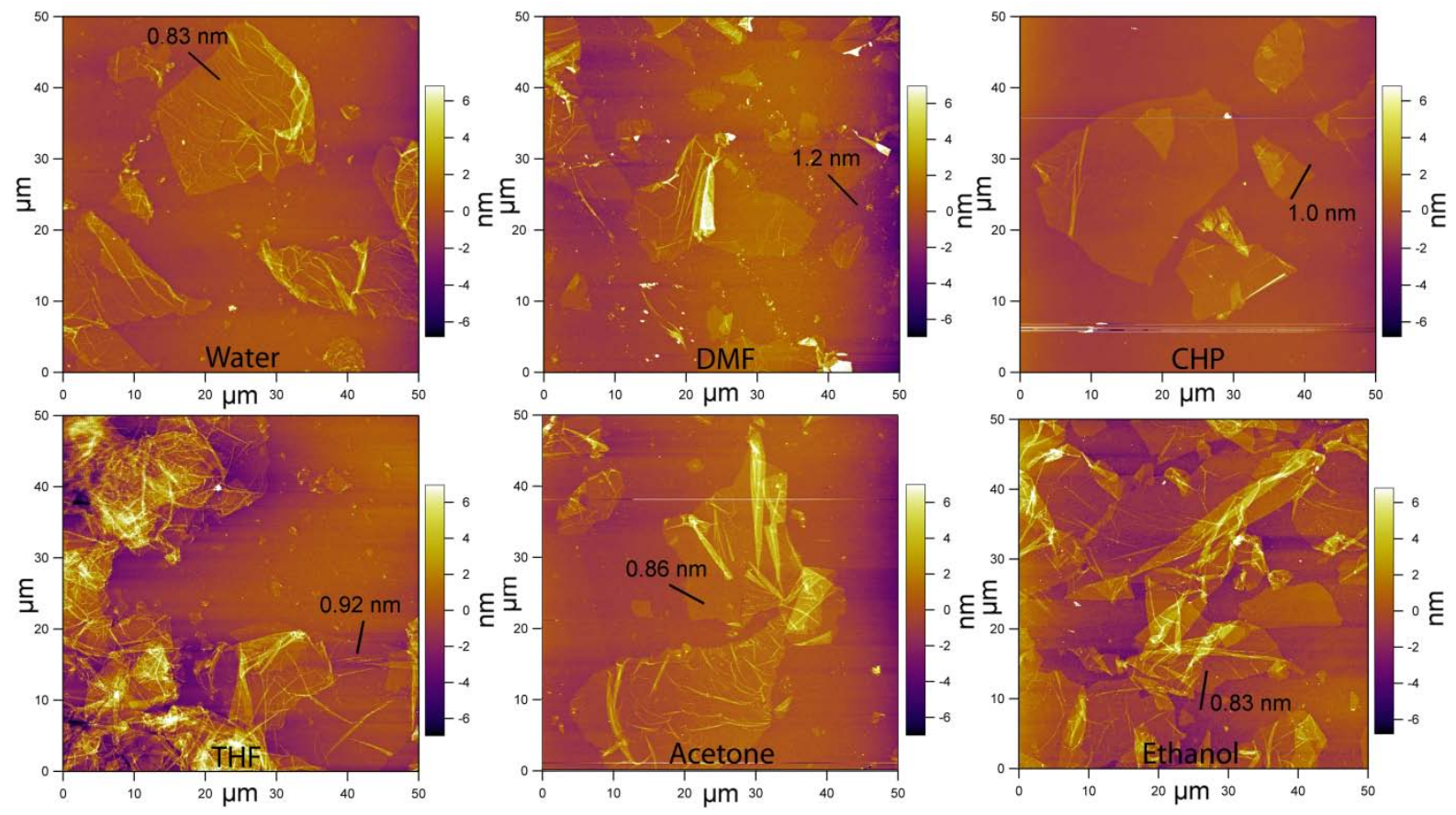

Figure 2. AFM images of GO sheets prepared from various organic solvent based LC GO dispersions. Marked line in each image shows the measured thickness of the sheet.

The change in the apparent thickness of GO sheets as a result of bonding with different solvents can also be confirmed by measuring the interlayer $d$-spacing of GO sheets in cast dried films made from various solvent based GO (Table 1). The $d$-spacing represents the interlayer distance between individual GO sheets in the paper material that are oriented perpendicularly to the diffraction plane. XRD measurements were performed to evaluate the effect of the solvents on the interlayer $d$-spacing of GO films (Figure 3a). The process of making GO in water or organic solvents is accompanied by an increase in the $d$-spacing between the graphene layers 
from about $0.34 \mathrm{~nm}$ to $\approx 0.8-1.1 \mathrm{~nm}$, which is related to the degree of oxidation and the hydration level (in the case of GO prepared in water) or the bonding of other solvent molecules to graphene sheets. ${ }^{1,23-26}$ Therefore, the peak in the XRD patterns of our as-prepared GO films corresponds to the layer-by-layer distance ( $d$-spacing) of each sample prepared in each organic solvent according to the Bragg's law. The position of the peak and correspondingly the $d$-spacing values observed in the XRD patterns varied with the solvent used. The differences on $d$-spacing values could be attributed to the confinement of organic solvent molecules in the lamellar GO sheet layers, as in the case of water for aqueous LC GO dispersions. This result could be illustrated by the larger $d$-spacings for GO sheets when dispersed in acetone, THF, CHP and DMF, which had intersheet distances of $0.978 \mathrm{~nm}, 1.01 \mathrm{~nm}$ and $1.17 \mathrm{~nm}$, respectively, than that of GO in water $(0.846 \mathrm{~nm})$. A very small shift ( $d$-spacing $0.820 \mathrm{~nm}$ ) was observed in ethanolbased LC GO consistent with the similarity of size with water. The measured $d$-spacing values were also in good agreement with the measured GO sheet thickness from AFM results (Table 1). Therefore, we suggest that the individual GO platelets are interlinked via a non-uniform network of hydrogen bonds mediated by oxygenated functional groups and solvent molecules.

Thermal gravimetric analysis (TGA) was also performed on GO papers (Figure 3b). GO papers which were cast from organic solvents with high boiling point temperatures $\left(154{ }^{\circ} \mathrm{C}\right.$ and $153{ }^{\circ} \mathrm{C}$ for CHP and DMF, respectively) lost considerably more weight at higher temperatures compared to GO recovered from water. In contrast, GO dispersed in volatile solvents (acetone, THF and ethanol) started to lose weight at much lower temperatures. This behavior can be attributed to the confinement of solvent molecules between GO sheets during film formation. The variation between the final weight losses was due to the different amount of solvents associated with each 
GO sample. The observed significant weight loss at around $180{ }^{\circ} \mathrm{C}$ to $200{ }^{\circ} \mathrm{C}$ is attributed to the partial reduction of GO.
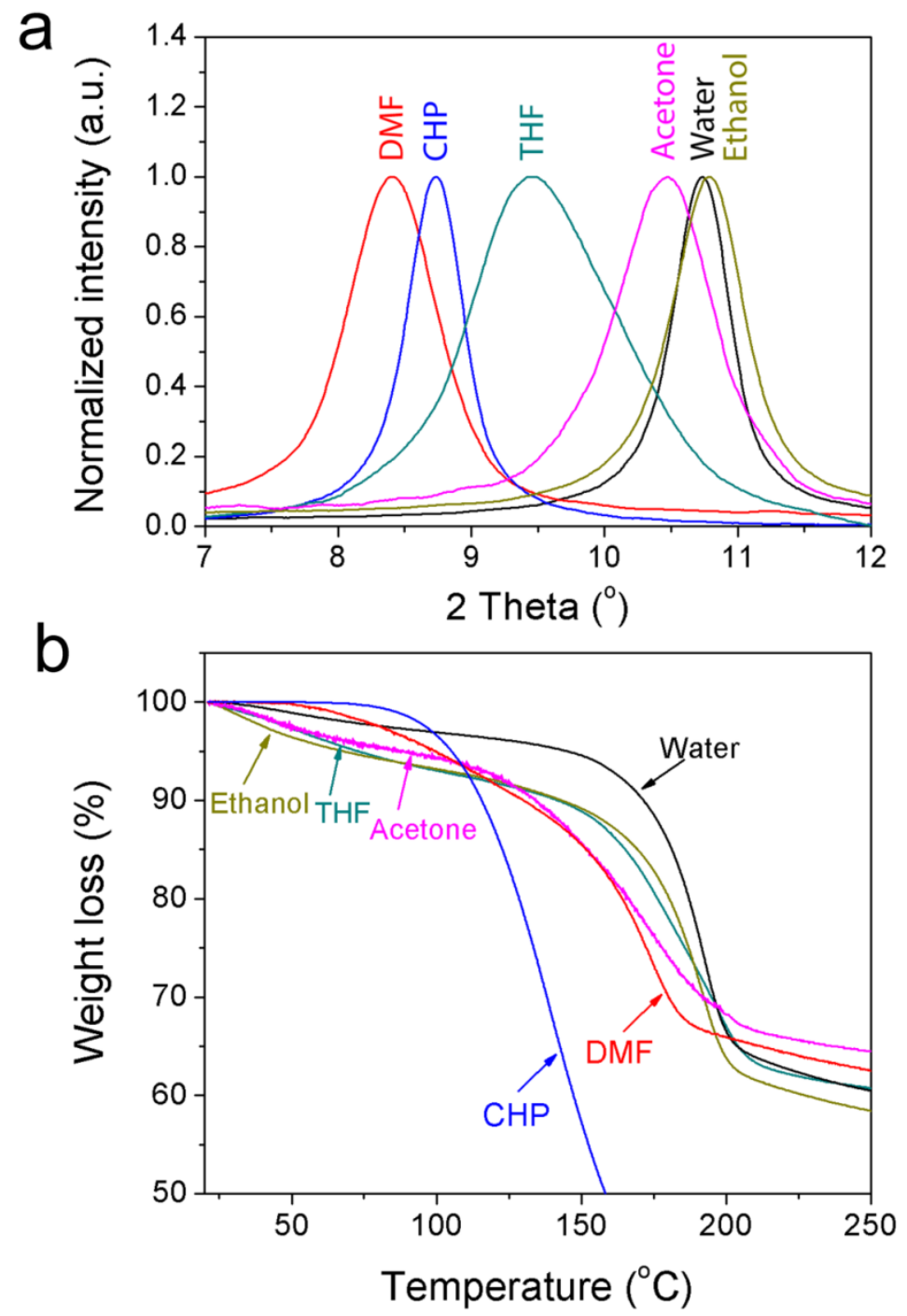

Figure 3. a) X-ray diffraction patterns (XRD) and b) Thermal gravimetric analysis (TGA) of GO films as a function of solvents.

\section{Insights into the formation of LC GO}

The self-assembly of amphiphiles in water is generally driven by hydrophobic interactions, ${ }^{23-}$ ${ }^{25}$ which is an important component of a larger solvophobic effect. ${ }^{23,26-28}$ Studies into the 
thermodynamic driving force for the self-assembly of amphiphiles into LC phases have highlighted that the solvophobic force is almost always dominated by entropic contribution. ${ }^{27,} 29$ This behavior is very similar to hydrophobic forces in water where the nature of these interactions is entropic in origin as the enthalpy change is actually unfavorable in most cases. ${ }^{30}$ Therefore, the process of self-assembly is an interplay of entropy and enthalpy terms in the free energy as given in Equation (1).

$$
\Delta \mathrm{G}^{\mathrm{o}} \text { self-assembly }=\Delta \mathrm{H}^{\mathrm{o}} \text { self-assembly }-\mathrm{T} \Delta \mathrm{S}^{\mathrm{o}}{ }_{\text {self-assembly }}
$$

Other contributing factors are hydrogen bonding, van der Waals interactions and electrostatic interactions. ${ }^{30}$ Graphene oxide is typically considered as a negatively charged amphiphilic molecule and as a result, ${ }^{1,22,31-32}$ the $\Delta \mathrm{G}^{\mathrm{o}}$ self-assembly in this case can be represented as the sum of the free energy change associated with hydrogen bonding, $\Delta \mathrm{G}_{\text {hydrogen bond, and electrostatic }}$ interactions, $\Delta \mathrm{G}_{\text {electrostatic }}$ (see Equation (2)).

$$
\Delta \mathrm{G}_{\text {self-assembly }}^{\mathrm{o}}=\Delta \mathrm{G}_{\text {hydrogen bond }}+\Delta \mathrm{G}_{\text {electrostatic }}
$$

With water as the self-assembly medium, both factors contribute to the free energy change term. Only a few other solvents other than water are capable of supporting amphiphilc selforganization. ${ }^{26,30,33-34}$ These solvents include a very limited range of multifunctional alcohols (such as ethylene glycol) and amides and a wide range of protic ionic liquids widely known as PILs. ${ }^{26,30,33-34}$ With PILs, where the solvent itself is an ion, the surface charge screening results in negligible electrostatic contribution, which is in contrast with water or organic solvents where the electrostatic charges play an important role in the free energy associated with ordering. ${ }^{30}$ In the case of LC GO, the solvent confined between the charged GO sheets adopts a more structural arrangement to balance the steric and repulsive forces. In this sense, GO can be considered as a self-assembling material due to the fact that it involves supermolecular and supramolecular 
interactions such as electrostatic repulsion between the adjacent sheets and hydrogen bonding with the confined solvent molecules, which compensate for the loss of rotational entropy during the self-assembly process. Therefore, in the case of organic solvents, it is the interplay between the ability to form multiple hydrogen bonds and electrostatic charges, manifested through Gordon parameter that governs the process of self-assembly.

The mechanism for the self-assembly process in organic solvents is considered to involve the solvophobic effect which is linked to the solvent cohesiveness. ${ }^{26}$ The Gordon parameter $\left(G=\gamma / V_{m}{ }^{1 / 3}\right)$ is a direct measure of the solvent cohesiveness where $\gamma$ is the surface tension and $V_{m}$ is the molar volume. ${ }^{35}$ High Gordon parameter represents firstly a higher chance to achieve selfassembled liquid crystalline phases and secondly a higher thermal stability. ${ }^{36}$ However, the ultra large size of GO sheets used in this study may push the boundaries toward organic solvents with Gordon parameters which are normally considered to be too low to be able to support solvophobic self-assembly. So far, the lowest reported Gordon parameter that supports amphiphilic self-assembly was $\mathrm{G}=0.576 \mathrm{~J} \mathrm{~m}^{-3}$ for a protic ionic liquid (EAB), ${ }^{34}$ which is far beyond the limit predicted by Evans $\left(\mathrm{G} \geq 1-1.2 \mathrm{~J} \mathrm{~m}^{-3}\right) .{ }^{37}$ However, as the Gordon parameter is directly dominated by surface tension and surface tension is a direct result of electrostatic charges and hydrogen bonding, in the case of PILs, the use of Gordon parameter might be irrelevant as the only dominating force is the ability of the liquid to form extensive hydrogen bonding network. Recently, Drummond et al. also discovered that some low molecular weight amides can be utilized as self-assembly media with a Gordon value as low as $0.53 \mathrm{~J} \mathrm{~m}^{-3}$, which is the direct result of the similarities between the chemical structure of PILs and the amides. ${ }^{26}$ However, apart from these two groups of solvents, no other organic polar solvent with lower Gordon parameter less than $1.3 \mathrm{~J} \mathrm{~m}^{-3}$ is yet found to act as an amphiphilic self-assembly medium. 
However, employing ultra large GO sheets has enabled us to achieve LC GO dispersions in a wide range of organic solvents with Gordon values previously deemed too low to support selfassembly. The list of solvents we have shown to support LC GO, along with Hansen parameter and Gordon parameter, are given in Table 2.

Table 2. Hansen parameters and Gordon parameter for the solvents could support LC GO.

\begin{tabular}{|c|c|c|c|c|c|c|c|}
\hline \multirow[t]{2}{*}{ Solvent } & \multirow{2}{*}{$\begin{array}{c}\begin{array}{l}\text { LC formation } \\
\text { concentration }\end{array} \\
\left(\mathrm{mg} \mathrm{ml}^{-1}\right)\end{array}$} & \multicolumn{4}{|c|}{ Hansen Parameter for solvents } & \multirow{2}{*}{$\begin{array}{l}\begin{array}{l}\text { Surface } \\
\text { tension }\end{array} \\
\left(\mathrm{mN} \mathrm{m}^{-1}\right)\end{array}$} & \multirow{2}{*}{$\begin{array}{c}\begin{array}{c}\text { Gordon } \\
\text { parameter }\end{array} \\
\left(\mathrm{J} \mathrm{m}^{-3}\right)\end{array}$} \\
\hline & & dispersive & polar & hydrogen & total & & \\
\hline Water & 0.25 & 15.5 & 16.0 & 42.3 & 47.8 & 72.8 & 2.77 \\
\hline ethylene glycol & 0.25 & 17.0 & 11.0 & 26.0 & 33.0 & 47.7 & 1.25 \\
\hline N-methyl pyrrolidone & 0.25 & 18.0 & 12.3 & 7.20 & 23.0 & 40.8 & 0.890 \\
\hline DMF & 0.25 & 17.4 & 13.7 & 11.3 & 24.9 & 37.0 & 0.869 \\
\hline dimethyl acetamide & 0.25 & 16.8 & 11.5 & 10.2 & 22.8 & 36.7 & 0.810 \\
\hline CHP & 0.25 & 18.2 & 6.80 & 6.50 & 20.5 & 42.3 & 0.770 \\
\hline methanol & 0.25 & 15.1 & 12.3 & 22.3 & 29.6 & 22.7 & 0.661 \\
\hline THF & 0.50 & 16.8 & 5.70 & 8.00 & 19.5 & 26.4 & 0.610 \\
\hline Acetone & 0.50 & 15.5 & 10.4 & 7.00 & 19.9 & 25.2 & 0.601 \\
\hline Ethanol & 0.25 & 15.8 & 8.80 & 19.4 & 26.5 & 22.1 & 0.569 \\
\hline isopropanol & 0.25 & 15.8 & 6.10 & 16.4 & 23.6 & 23.0 & 0.542 \\
\hline
\end{tabular}

Apart from water which has a Gordon parameter higher than $1.2 \mathrm{Jm}^{-3}$, DMF (which is an amide) shares a degree of structural similarity with PILs and can form extensive hydrogen bonded networks similar to water. ${ }^{34}$ Therefore, although the Gordon parameter is very low, DMF could afford formation of LC GO. For the case of other solvents, it is evident that the 
ability to support LC GO is largely governed by the capability of the solvents to form hydrogen bonds. As an example, although the Gordon value of isopropanol and ethanol is very low ( $\mathrm{G}=$ $0.541 \mathrm{~J} \mathrm{~m}^{-3}$ and $\mathrm{G}=0.569 \mathrm{~J} \mathrm{~m}^{-3}$, respectively), their ability to form multiple hydrogen bonds which is even much higher than DMF, can overcome the low cohesive energy density of the solvent. On the other hand, the lower ability of acetone and THF to form hydrogen bonds resulted in an increased GO concentration required for $\mathrm{LC}$ formation $\left(0.50 \mathrm{mg} \mathrm{ml}^{-1}\right)$ compared to ethanol $\left(0.25 \mathrm{mg} \mathrm{ml}^{-1}\right)$, which has slightly lower Gordon parameter but much higher hydrogen bonding ability. As a result, it is safe to assume that the ability to form an extensive hydrogen bonding network is the most important parameter to govern the self-organization process in the case of LC GO. Consequently, the interplay between the hydrogen bonding and the Gordon parameter can effectively determine: (i) whether an organic solvent can induce the self-assembly process, and (ii) the lowest possible concentration in which spontaneous self-organization can occur.

\section{Exploitation of the self-assembly nature of LC GO}

The intrinsic self-assembly nature of LC materials can be used to exploit them as versatile templates for the synthesis and alignment of nanoparticles. ${ }^{11,14}$ We utilized LC GO to induce liquid crystallinity to SWNTs dispersion through the addition of LC GO to the SWNTs dispersion. Here we have investigated the effect of introducing SWNTs to LC GO dispersion. POM micrographs of LC GO-SWNTs are presented at Figures 4a and 4b which show the nematic LC behavior of as-prepared mixtures in CHP and DMF, respectively. Although SWNTs and rod shaped particles under some specific conditions can form LC phases, this requires

modification of the SWNTs surface by bio-polymers, ${ }^{38-39}$ functionalization (with a subsequent compromise in electronic properties), ${ }^{40-41}$ or the use of superacids. ${ }^{42-43}$ Here we have dispersed 
SWNTs in CHP at concentrations as high as surfactant assisted dispersions $\left(1 \mathrm{mg} \mathrm{ml}^{-1}\right){ }^{20}$ Subsequent mixing of this SWNT dispersion with CHP- or DMF-based lyotropic LC GO resulted in birefringence yet preserves the fundamental properties of SWNTs (no dispersant was added). It has been shown that organic solvent stabilized SWNT dispersions are sensitive to the presence of water whereby the addition of very small amounts of water will cause the dispersion to agglomerate and crash out of solution. ${ }^{44}$ The water free nature of our as-prepared LC GO in organic solvents is demonstrated by the fact that SWNTs do not agglomerate and crash out of the dispersion when they are added to the as-prepared LC GO in organic solvents. The quality of the dispersion even after addition of the GO is evidenced by the UV Vis spectra, (Figure 4c). The well-resolved inter-band transitions in the UV/Vis-near-IR spectra of the SWNT dispersion before and after the addition of LC GO indicate that SWNT sizes are preserved in the composite formulation. According to Smalley and Hague, ${ }^{45}$ UV/Vis-near-IR spectroscopy is the most reliable method to determine SWNTs size distribution based on Van Hove singularities. As it is evident from UV/Vis-near-IR spectra, the size of our nanotubes is predominantly in the range of $1 \mathrm{~nm}$ and $0.95 \mathrm{~nm}$ corresponding to the wavelength of $\sim 1300 \mathrm{~nm}$ and $\sim 1150 \mathrm{~nm}$ in the asprepared SWNTs dispersions, respectively. The quality of SWNTs dispersion (bundle size) was also preserved during the combination with LC GO.

It is pertinent to mention that although many particles can disturb the liquid crystal director field depending on the particle size, shape and surface interaction with liquid crystal media, ${ }^{46} \mathrm{GO}$ in this regard enjoys a unique benefit. This unique benefit is due to minimizing the exert forces or torques in the direction perpendicular to the director to promote a configuration in which the distortion is minimal, leading to the in-plane alignment of anisotropic particles (the ordering of particles on GO planes) such as SWNTs; as evident from the strength of our as-prepared 
composite films (see next section). Prior to this work, ${ }^{47-49}$ the only successful method of increasing the CNT concentration in lyotropic LCs has been through using extensive amounts of surfactants (which can adversely CNTs performance). ${ }^{50}$ Only trace amounts of CNTs have been dispersed in thermotropic LCs in order to avoid CNT aggregation. ${ }^{47,49}$ However, in our case, we have been able to disperse and organize substantial amounts of SWNTs (up to $10 \mathrm{wt} \%$ ) in GO LC without losing birefringence properties and observing any aggregation. Therefore, this LC formulation was utilized for a facile fabrication of self-assembled layer-by-layer LC GO-SWNT 3D assemblies. 

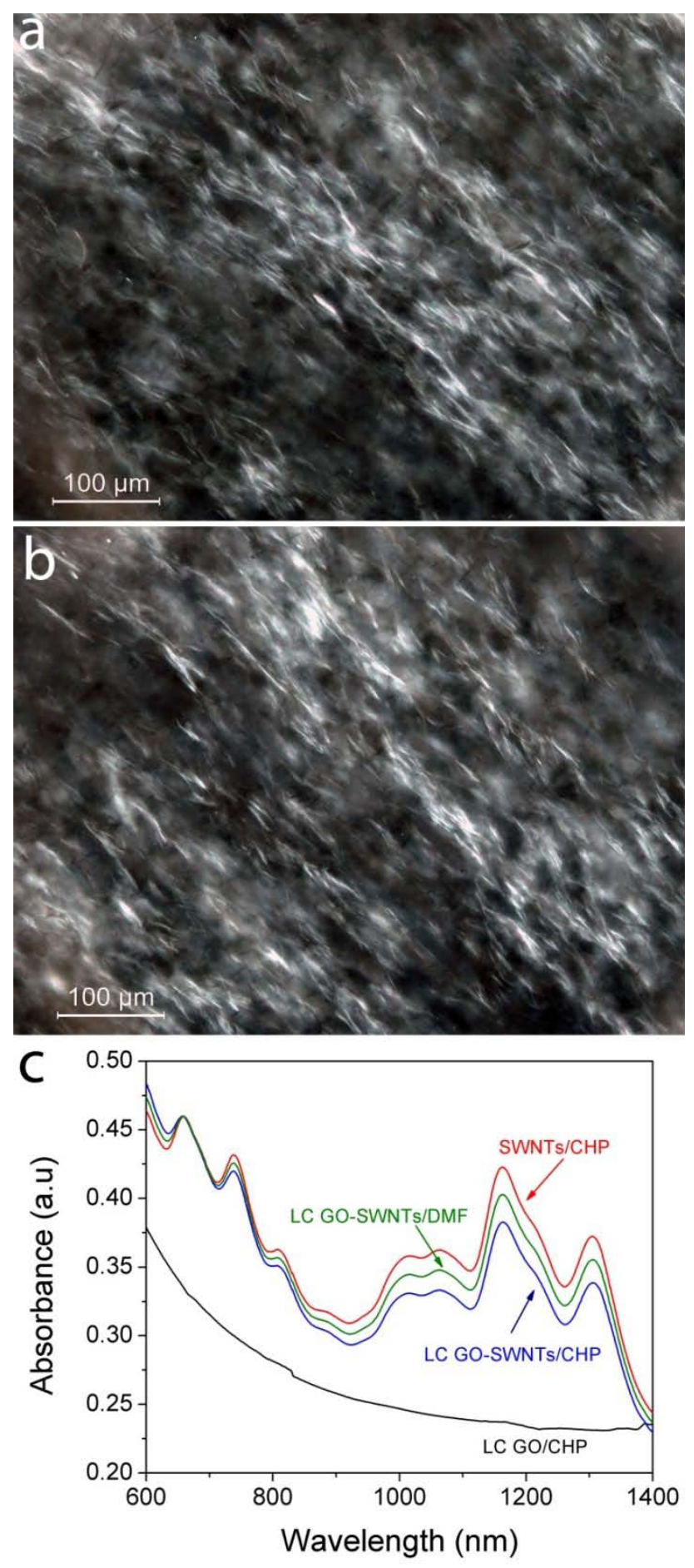

Figure 4. Representative POM micrographs of a) LC GO-SWNTs/CHP (90:10 at 1 $\mathrm{mg} \mathrm{ml}^{-1}$ ), b) LC GO-SWNTs/DMF (90:10 at $\sim 1 \mathrm{mg} \mathrm{ml}^{-1}$ ). c) UV/Vis-near-IR spectra of SWNTs and LC GO dispersions before and after mixing together. 


\section{Self-assembled layer-by-layer multifunctional composite}

The LC properties of our as-prepared LC GO-SWNTs dispersions in organic solvents induce a spontaneous self-assembly into engineered long range ordered layer-by-layer 3D structures upon simple casting and drying as shown in Figure 5. The ease of synthesis, much shorter processing time and high scalability of this route in contrast with other layer-by-layer production methods (such as Langmuir-Blodgett deposition) offers the opportunity for facile fabrication of 3D frameworks with exceptional properties. The excluded volume generated by large GO sheets for SWNTs results in an entropic rearrangement to form long range ordering. Therefore, LC GO in CHP can be employed as a dispersing media to process SWNTs based on hydrophobic and $\pi-\pi$ interactions as well as a medium in which SWNTs can be self-assembled. This can consequently result in the fabrication of highly aligned and macroscopically periodic self-assembled structures of GO-SWNTs hybrid material. The aggregate-free nature of our as-prepared LC GO-SWNTs enabled us to achieve ultra-stiff, ultra-strong layer-by-layer 3D architecture with high elongationat-break which enjoy an average modulus of $51.3 \mathrm{GPa}$, tensile strength of $505 \mathrm{MPa}$ and elongation at break of 9.8\% (Figure 6), which is much higher than the parent GO architecture. 

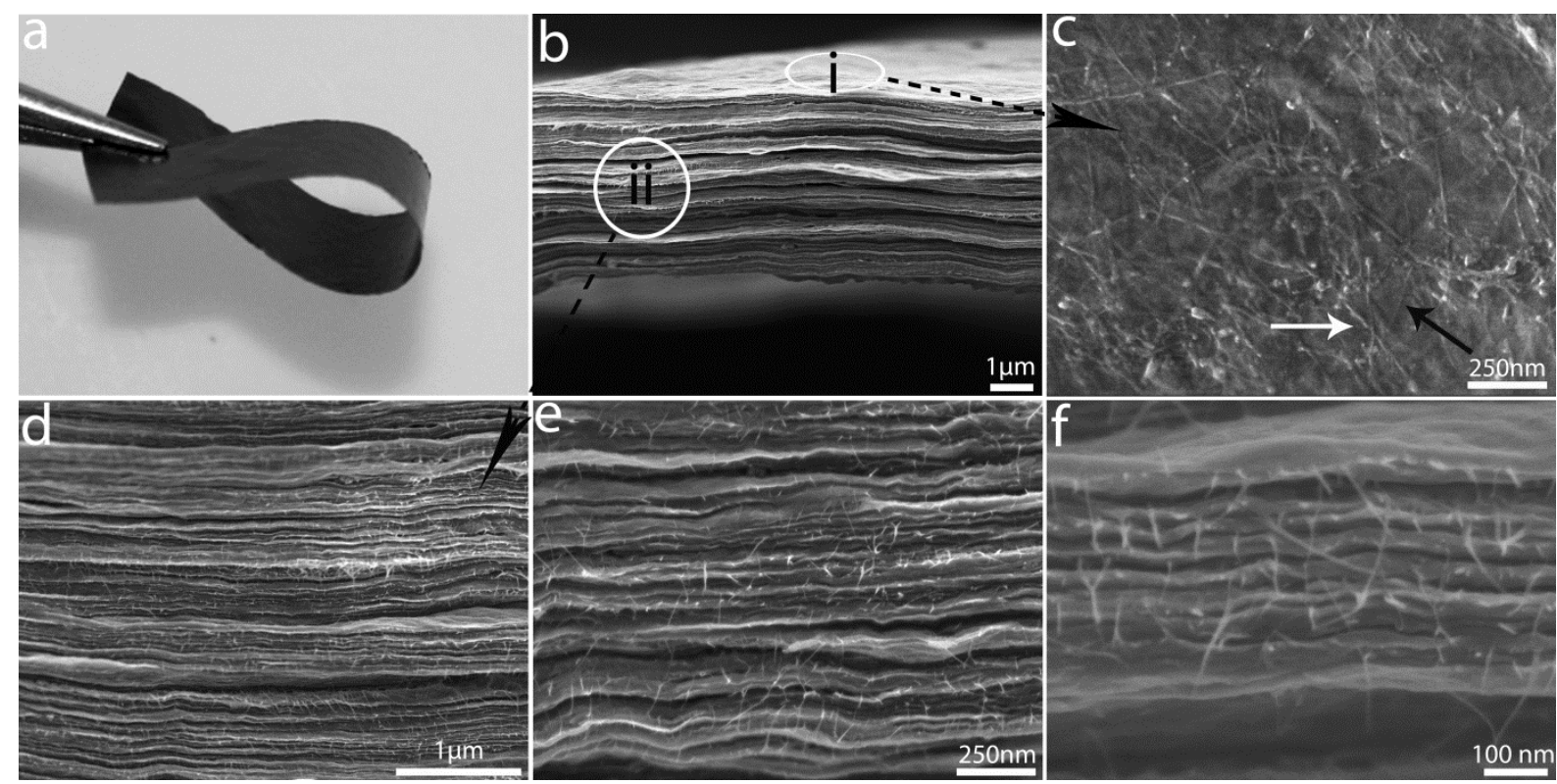

Figure 5. a) Representative photograph of a flexible free-standing paper of LC GO-SWNT made by cast drying method. b) SEM image of the cross-section of as-cast dried LC GO-SWNT paper. c) SEM image of the surface of the layer-by-layer composite which is marked as region (i) in (b). Some of the SWNTs are laid on the surface of the paper (white arrow) while others are placed between layers of GO sheet (black arrow). Transparency of the monolayer/few layers of GO sheets allows observing tubes sites in different layers. d-f) Cross-section of composite paper at different magnifications (marked as (ii) in (b)), confirmed the self-oriented nature of the composite as well as maintaining SWNTs debundled after the fabrication of composite. 

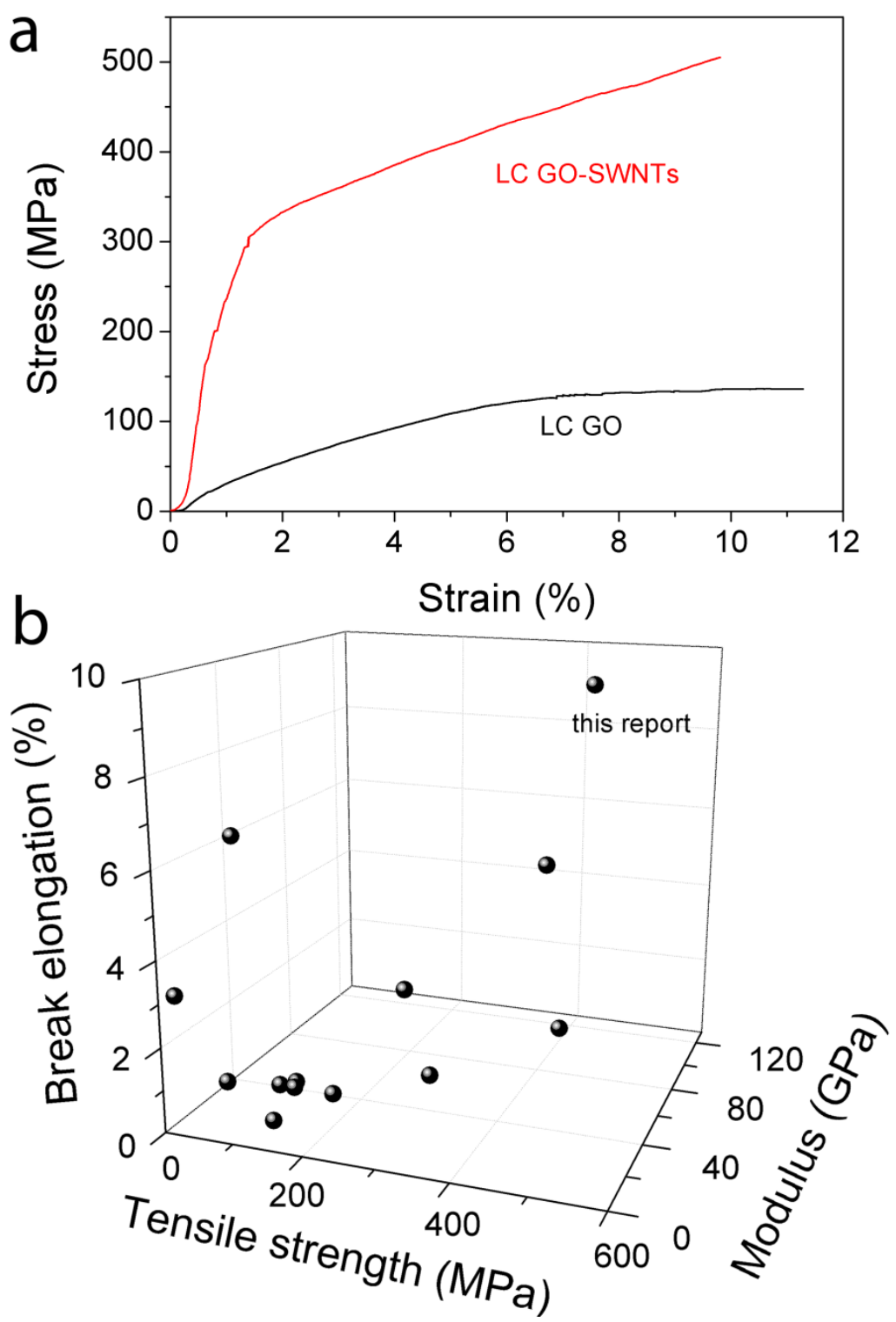

Figure 6. a) Stress-strain curves of LC GO and LC GO-SWNTs self-assembled composite. b) Diagram of mechanical performance data for layer-by-layer nanocomposites and carbon-based neat papers andfibers in selected significant published reports and in this study (see also table S1). 
The average strength, reported here, is much higher than those reported for bucky papers, ${ }^{51}$ GO and rGO papers (prepared by either filtration or casting strategies) and fibers (either as is or cross-linked), ${ }^{17,52-55}$ paper-like materials based on vermiculite, ${ }^{56}$ flexible graphite foil, ${ }^{57-58}$ neat SWNTs fibers, ${ }^{42-43,55,59-60}$ and even layer-by-layer assembled polymer, ${ }^{61}$ SWNTs, ${ }^{62}$ or MWNTs nanocomposites. ${ }^{63}$ As proposed by Ruoff et al. very high mechanical strength can be obtained if ordering and alignment of fibrils/macromolecules are achieved. ${ }^{52}$ In comparison with irregularly laid-down individual fibrils obtained via filtration, the LC route provided us with a self-mediated platform to organize and order SWNTs resulting in an extraordinarily high mechanical strength. Our average Modulus is also considerably higher than all those (Figure $6 \mathrm{~b}$ and Table S1) and just inferior to either SWNT fibers composites with polymers or extensively processed SWNTs. 43, 55, 64 The as-prepared composite paper exhibited an exceptional conductivity of $1500 \mathrm{~S} \mathrm{~m}^{-1}$.

The ultra-large nature of our as-prepared GO sheets (with lateral size in excess of $32 \mu \mathrm{m}$ compared to typically few hundreds of nm in the case of other reports) provides us with a highly wrinkled topography, ${ }^{1,65}$ which can first contribute to an overall increase in strain, and second maximize the fraction of the surface area available for mechanical reinforcement and toughening. ${ }^{52,55}$ Therefore, in the case of ultra large GO sheets, the initial straightening due to wrinkled topography plays a crucial role in the observed increase in elongation-at-break of our as prepared GO compared to other reports. Furthermore, this enhanced wrinkled topography provides us with a unique platform to accommodate individual, separated SWNTs in between the GO sheets resulting in an overall enhancement in exploitation of the extraordinary mechanical properties of SWNTs. Also, the introduction of SWNTs can prevent the restacking of individual two-dimensional GO sheets, further enhancing the properties of the hybrid material. 
Furthermore, our GO-SWNTs hybrid material exhibited a very high toughness in the order of 20 $\mathrm{J} \mathrm{g}^{-1}$, which is almost two orders of magnitude higher than GO paper, ${ }^{52}$ three orders of magnitude higher than pristine bucky paper, ${ }^{51,66}$ and flexible graphite foils and even 7 times higher than GO/rGO fibers. ${ }^{17}$ The flat fracture surface of our ruptured paper is evidence of the good material homogeneity and layer-by-layer nature of both our as-prepared neat and hybrid papers. The strong interfacial bonding in the case of our hybrid paper is also evident from the straight fracture surface in contrast to the rupture of bucky paper (Figure 5). In regards to mechanical properties, SWNTs act as bridging components between individual GO sheets (see high magnification SEM micrographs in Figure 5d-f). As the GO sheets are not strongly attached together, reinforcing them with SWNTs can result in an overall increase in mechanical properties. SWNTs can bridge individual GO sheets and therefore increase the shear force between GO sheets. Furthermore, the most important limitation of using SWNTs as reinforcing agents is the intertube and interfacial slippage within bundles. The poor load transfer within bundles results in interfacial slippage as the effective moduli and strength for bundles are far below those expected for individual SWNTs. The individual nature of SWNTs reported in our study, as evident in high resolution SEM figures presented in Figure 5 and the well-resolved inter-band transitions in the UV/Vis-near-IR spectra of the SWNT dispersion before and after the addition of LC GO presented in Figure 4, ensures that the shear slippage of nanotubes within the bundle does not occur and the mechanical properties of SWNTs used in this study are preserved resulting in an overall enhancement of the final composite properties. The findings presented here will pave the way to versatile and highly scalable routes for the fabrication of a wide range of large-scale 3D graphene based architectures (including metallic or polymer based composites) 
with extensive applications in multifunctional wearables, sensors, supercapacitor devices and electronic gadgets.

\section{Conclusion}

In summary, our discovery of lyotropic LC GO dispersions in a range of organic solvents contributes to the fundamental understanding of the solvophobic effect and the parameters affecting the self-assembly process. The ability of the solvents to promote self-assembly in GO is governed by their polarity and is linked to the ability to form extensive hydrogen bonding. The steric hindrance between the highly charged GO sheets is the factor that overcomes the unfavorable loss of rotational entropy associated with ordering. The expansion of the list of known solvents that can promote the self-assembly process and lyotropic liquid crystallinity has enabled us to tailor-make processable self-assembled, self-oriented SWNTs/GO hybrid composites with superior mechanical performances (Table S1). This discovery could provide practical solutions to the processability of a wide range of materials that require organic solvents because of solubility issues and/or water sensitivity (i.e. metal oxides, polymers and nanomaterials).

\section{Methods}

LC GO synthesis: In order to obtain fully oxidized graphite and preserve the high initial lateral sizes of graphite flakes, dry expandable graphite flakes (3772, Asbury Graphite Mills USA) were first thermally treated at $1050{ }^{\circ} \mathrm{C}$ for 15 sec. The resultant expanded graphite (EG) was used as the precursor for GO synthesis following previously described methods. ${ }^{1,65,67}$ Briefly, $5 \mathrm{~g}$ of EG and 1 lit of sulfuric acid were mixed and stirred in a flask for 24h. $50 \mathrm{~g}$ of $\mathrm{KMnO}_{4}$ was added to 
the mixture dropwise. The mixture was transferred into an ice bath, and 1 lit of Milli-Q water and $250 \mathrm{~mL}$ of $\mathrm{H}_{2} \mathrm{O}_{2}$ were poured slowly into the mixture, realizing a colour change of the suspension to light brown. Having stirred for another $30 \mathrm{~min}$, the GO particles were then washed and centrifuged with a $\mathrm{HCl}$ solution (9:1 water: $\mathrm{HCl}$ by volume), then centrifuged again and washed with Milli-Q water until the $\mathrm{pH}$ of the solution became about 5 to 6 . The resultant ultralarge GO sheets were dispersed in deionized water by gentle shaking (i.e. without the aid of sonication process).

N-N-dimethylformamide (DMF), N-Cyclohexyl-2-pyrrolidone (CHP), tetrahydrofuran (THF), acetone, ethylene glycol, N-methyl pyrrolidone (NMP), dimethyl acetamide (DMAc), methanol, isopropanol and absolute ethanol, all from Sigma, have been chosen for investigation. LC GO dispersions in various organic solvents were prepared by extraction of water from the parent aqueous LC GO dispersion via repeated centrifugation-washing steps (6 times of 10 to 30 minutes at $11000 \mathrm{rpm}$, ProSciTech TG16WS) using the selected solvent. Briefly, $15 \mathrm{ml}$ of the parent aqueous LC GO (2.5 mg ml-1) was poured into a $50 \mathrm{ml}$ centrifuge tube (Nalgene centrifuge tube) to which $20 \mathrm{ml}$ of the selected solvent was added and then mixed vigorously by vortex shaking. After centrifugation, $30 \mathrm{ml}$ of the supernatant was extracted, replaced with $30 \mathrm{ml}$ of the selected solvent and then mixed vigorously by vortex shaking. This process was repeated 5 times to replace the water with the selected organic solvent.

Layer-by-layer self-assembly of the LC GO-SWNT composite: SWNTs dispersion was prepared by adding $15 \mathrm{mg}$ of HiPCO-SWNTs to $15 \mathrm{ml}$ of CHP. This dispersion was subjected to a $3 \mathrm{hr}$ of high-power tip sonication (SONICS Vibra Cell $500 \mathrm{~W}, 30 \%$ amplitude) followed by a one day low-power bath sonication (Branson B5500R-DTH). The SWNTs/CHP (1 mg ml ${ }^{-1}$ ) dispersion was then mixed with LC GO in DMF or in CHP $\left(2.5 \mathrm{mg} \mathrm{ml}^{-1}\right)$ at the weight ratio of 
(10:90) followed by $10 \mathrm{~min}$ of vortex mixing. A self-assembled layer-by-layer composite was fabricated by casting $2 \mathrm{ml}$ of the composite formulation into a Teflon mold $(2 \mathrm{~cm} \mathrm{X} 2 \mathrm{~cm})$ and then oven dried at $110{ }^{\circ} \mathrm{C}$ for 2 days. The resultant oven-dried GO-SWNT paper was washed several times by DMF, acetone and dried in air to remove the remaining solvent. GO paper, as control, was made using GO in DMF; however, SWNT in CHP was not form free standing paper using simple casting.

Characterizations: AFM analysis was carried out by first depositing GO sheets from their dispersions on pre-cleaned and silanized silicon wafer (300 nm SiO 2 layer). Silane solution was prepared by mixing 3-Aminopropyltriethoxysilane (Sigma) with water (1:9 vol) and one drop of hydrochloric acid (Sigma). Pre-cut silicon substrates were silanized by immersing them in aqueous silane solution for $30 \mathrm{~min}$ and then washed thoroughly with Millipore water. LC GO in each solvent was first diluted to $\sim 50 \mu \mathrm{g} \mathrm{ml}^{-1}$, then GO sheets were deposited onto silanized silicon substrates by immersing a silicon substrate into the GO dispersion for 5 seconds, then immersed in the solvent bath (the same solvent used to form the LC GO) for 30 seconds and then dried under nitrogen flow at room temperature. The CHP based sample was then heated up to $70^{\circ} \mathrm{C}$ to be dried due to its higher boiling point compared to the other solvents. Prior to AFM analysis, GO sheets were observed under an optical microscope to ensure uniform GO sheet deposition was achieved. Atomic force microscopy (MFP-3D AFM Asylum Research, CA) was carried out in tapping mode under ambient conditions. X-ray diffraction (XRD) studies were performed using a powder XRD system (Philips 1825) with $\mathrm{CuK} \alpha$ radiation $(\lambda=0.154 \mathrm{~nm})$ operating at $40 \mathrm{keV}$ and with a cathode current of $20 \mathrm{~mA}$. Thermal gravimetric analyses (TGA) were carried out in nitrogen atmosphere from room temperature to $250{ }^{\circ} \mathrm{C}$ at a temperature ramp rate of $1{ }^{\circ} \mathrm{C} \min ^{-1}$. The birefringence of LC GO dispersions was examined by polarized optical 
microscopy (POM,Leica CTR 6000) operated in transmission mode by looking at a drop of LC GO on a glass slide. UV/Vis-near-IR spectra were recorded using a Shimadzu UV-3600 spectrometer from $600 \mathrm{~nm}$ to $1400 \mathrm{~nm}$. The conductivity of LC GO-SWNTs paper was measured using a JANDEL 4-point probe resistivity system (Model RM2) with a linear four-point probe head. The mechanical properties of the composite paper were measured using a Shimadzu tensile tester (EZ-S) at a strain rate of $0.5 \% \mathrm{~min}^{-1}$ parallel to the GO plain in the paper. Youngs modulus $(\mathrm{Y})$, tensile strength $(\sigma)$, breaking strain $(\varepsilon)$, and breaking energy (toughness) were calculated, and the average reported for 10 samples. The thickness of the composite papers was $\sim 10 \mu \mathrm{m}$ and the papers are cut in $20 \mathrm{~mm}$ by $5 \mathrm{~mm}$. The obtained strips were mounted on aperture cards with commercial superglue and allowed to air dry. Note, only $10 \mathrm{~mm}$ of each sample was exposed to the applied strain as $5 \mathrm{~mm}$ from each side is used for gluing to the aperture card.

\section{ACKNOWLEDGMENT}

The authors thank the discussions and guidance from A/Prof. Chee O Too. The authors thank Tony Romeo for technical assistance, the Australian Research Council (ARC) for financial support, and the ANFF Materials Node for their provision of research facilities. This work was supported by ARC Discovery Project DP1093952 (KK and SHA) and ARC Federation Fellowship (GGW). Expandable graphite was kindly provided by Asbury Carbons.

\section{ABBREVIATIONS}

liquid crystalline, LC; graphene oxide, GO; single walled carbon nanotubes, SWNTs; tetrahydrofuran, THF; N-dimethylformamide, DMF; N-cyclohexyl-2-pyrrolidone, CHP; Nmethyl pyrrolidone, NMP; dimethyl acetamide; DMAc; polarized optical microscopy, POM; Atomic force microscopy, AFM; X-ray diffraction, XRD; Thermal gravimetric analysis, TGA. 
ASSOCIATED CONTENT

AUTHOR INFORMATION

\section{Corresponding Author}

*To whom correspondence should be addressed. Email: gwallace@uow.edu.au

\section{Author Contributions}

¥These authors contributed equally to this work.

The manuscript was written through contributions of all authors. All authors have given approval to the final version of the manuscript.

\section{Funding Sources}

The authors thank the Australian Research Council (ARC) for financial support and the ANFF Materials Node for their provision of research facilities. This work was supported by ARC Discovery Project DP1093952 (KK and SHA), ARC Queen Elizabeth II Fellowship (SEM) and

\section{Supporting Information.}

Calculation of the theoretical concentration for LC formation. Figure S1 to S6, representative polarized optical micrographs of LC GO dispersions in different solvents at various GO concentrations. Figure S7 XRD pattern of LC GO/SWNTs composite. Table S1, comparison of LC GO/ SWNTs mechanical performance data with layer-by-layer nanocomposites and carbon- 
based neat papers anfibers in selected significant previous reports and in this study. This material is available free of charge via the Internet at http://pubs.acs.org.

\section{REFERENCES}

1. Aboutalebi, S. H.; Gudarzi, M. M.; Zheng, Q. B.; Kim, J.-K., Spontaneous Formation of Liquid Crystals in Ultralarge Graphene Oxide Dispersions. Adv. Funct. Mater. 2011, 21, 29782988.

2. Dan, B.; Behabtu, N.; Martinez, A.; Evans, J. S.; Kosynkin, D. V.; Tour, J. M.; Pasquali, M.; Smalyukh, I. I., Liquid Crystals of Aqueous, Giant Graphene Oxide Flakes. Soft Matter 2011, 7, 11154-11159.

3. Guo, F.; Kim, F.; Han, T. H.; Shenoy, V. B.; Huang, J.; Hurt, R. H., Hydration-Responsive Folding and Unfolding in Graphene Oxide Liquid Crystal Phases. ACS Nano 2011, 5, 80198025.

4. Kim, J. E.; Han, T. H.; Lee, S. H.; Kim, J. Y.; Ahn, C. W.; Yun, J. M.; Kim, S. O., Graphene Oxide Liquid Crystals. Angew. Chem. Int. Ed. 2011, 50, 3043-3047.

5. Eda, G.; Chhowalla, M., Graphene Patchwork. ACS Nano 2011, 5, 4265-4268.

6. Xu, Z.; Gao, C., Aqueous Liquid Crystals of Graphene Oxide. ACS Nano 2011, 5, 2908-2915.

7. Behabtu, N.; Lomeda, J. R.; Green, M. J.; Higginbotham, A. L.; Sinitskii, A.; Kosynkin, D. V.; Tsentalovich, D.; Parra-Vasquez, A. N. G.; Schmidt, J.; Kesselman, E., et al., Spontaneous High-Concentration Dispersions and Liquid Crystals of Graphene. Nat. Nanotechnol. 2010, 5, 406-411.

8. Hu, X.; Xu, Z.; Gao, C., Multifunctional, Supramolecular, Continuous Artificial Nacre Fibres. Sci. Rep. 2012, 2.

9. Zamora-Ledezma, C.; Puech, N.; Zakri, C.; Grelet, E.; Moulton, S. E.; Wallace, G. G.; Gambhir, S.; Blanc, C.; Anglaret, E.; Poulin, P., Liquid Crystallinity and Dimensions of Surfactant-Stabilized Sheets of Reduced Graphene Oxide. J. Phys. Chem. Lett. 2012, 3, 24252430 .

10. Fong, C.; Le, T.; Drummond, C. J., Lyotropic Liquid Crystal Engineering-Ordered Nanostructured Small Molecule Amphiphile Self-Assembly Materials by Design. Chemical Society Reviews 2012, 41, 1297-1322.

11. Bisoyi, H. K.; Kumar, S., Liquid-Crystal Nanoscience: An Emerging Avenue of Soft SelfAssembly. Chemical Society Reviews 2011, 40, 306-319.

12. Hamley, I. W., Nanotechnology with Soft Materials. Angewandte Chemie International Edition 2003, 42, 1692-1712.

13. Lagerwall, J. P. F.; Scalia, G., A New Era for Liquid Crystal Research: Applications of Liquid Crystals in Soft Matter Nano-, Bio- and Microtechnology. Current Applied Physics 2012, 12, 1387-1412.

14. Dellinger, T. M.; Braun, P. V., Lyotropic Liquid Crystals as Nanoreactors for Nanoparticle Synthesis. Chemistry of Materials 2004, 16, 2201-2207.

15. Kijima, T.; Yoshimura, T.; Uota, M.; Ikeda, T.; Fujikawa, D.; Mouri, S.; Uoyama, S., NobleMetal Nanotubes (Pt, Pd, Ag) from Lyotropic Mixed-Surfactant Liquid-Crystal Templates. Angewandte Chemie International Edition 2004, 43, 228-232. 
16. Hulvat, J. F.; Stupp, S. I., Anisotropic Properties of Conducting Polymers Prepared by Liquid Crystal Templating. Advanced Materials 2004, 16, 589-592.

17. Xu, Z.; Gao, C., Graphene Chiral Liquid Crystals and Macroscopic Assembled Fibres. Nat Commun. 2011, 2, 571.

18. Yousefi, N.; Gudarzi, M. M.; Zheng, Q.; Aboutalebi, S. H.; Sharif, F.; Kim, J.-K., SelfAlignment and High Electrical Conductivity of Ultralarge Graphene Oxide-Polyurethane Nanocomposites. J. Mater. Chem. 2012, 22, 12709-12717.

19. Krishnan, D.; Kim, F.; Luo, J.; Cruz-Silva, R.; Cote, L. J.; Jang, H. D.; Huang, J., Energetic Graphene Oxide: Challenges and Opportunities. Nano Today 2012, 7, 137-152.

20. Bergin, S. D.; Sun, Z.; Streich, P.; Hamilton, J.; Coleman, J. N., New Solvents for Nanotubes: Approaching the Dispersibility of Surfactants. J. Phys. Chem. C 2009, 114, 231-237. 21. Dimiev, A. M.; Alemany, L. B.; Tour, J. M., Graphene Oxide. Origin of Acidity, Its Instability in Water, and a New Dynamic Structural Model. ACS Nano 2013, 7, 576-588.

22. Medhekar, N. V.; Ramasubramaniam, A.; Ruoff, R. S.; Shenoy, V. B., Hydrogen Bond Networks in Graphene Oxide Composite Paper: Structure and Mechanical Properties. ACS Nano 2010, 4, 2300-2306.

23. Southall, N. T.; Dill, K. A.; Haymet, A. D. J., A View of the Hydrophobic Effect. The Journal of Physical Chemistry B 2001, 106, 521-533.

24. Chandler, D., Interfaces and the Driving Force of Hydrophobic Assembly. Nature 2005, 437, 640-647.

25. Meyer Emily E.; Rosenberg Kenneth J.; Israelachvili Jacob, Recent Progress in Understanding Hydrophobic Interactions. PANS 2006, 103, 8.

26. Greaves, T. L.; Weerawardena, A.; Drummond, C. J., Nanostructure and Amphiphile SelfAssembly in Polar Molecular Solvents: Amides and the "Solvophobic Effect". Physical Chemistry Chemical Physics 2011, 13, 9180-9186.

27. Ray, A., Solvophobic Interactions and Micelle Formation in Structure Forming Nonaqueous Solvents. Nature 1971, 231, 313-315.

28. Akhter, M. S., Studies on Solvophobic Interactions and Micelle Formation in Non Aqueous Solvents. Colloids and Surfaces A: Physicochemical and Engineering Aspects 1999, 150, 25-30.

29. Evans, D. F.; Yamauchi, A.; Roman, R.; Casassa, E. Z., Micelle Formation in Ethylammonium Nitrate, a Low-Melting Fused Salt. Journal of Colloid and Interface Science 1982, 88, 89-96.

30. Greaves, T. L.; Drummond, C. J., Ionic Liquids as Amphiphile Self-Assembly Media. Chemical Society Reviews 2008, 37, 1709-1726.

31. Withers, N., Graphene Oxide: Surfactant Sheets. Nat Chem 2010.

32. Kim, J.; Cote, L. J.; Kim, F.; Yuan, W.; Shull, K. R.; Huang, J., Graphene Oxide Sheets at Interfaces. J. Am. Chem. Soc. 2010, 132, 8180-8186.

33. Ray, A., Micelle Formation in Pure Ethylene Glycol. Journal of the American Chemical Society 1969, 91, 6511-6512.

34. Greaves, T. L.; Weerawardena, A.; Fong, C.; Drummond, C. J., Many Protic Ionic Liquids Mediate Hydrocarbon-Solvent Interactions and Promote Amphiphile Self-Assembly. Langmuir 2006, 23, 402-404.

35. Evans, D. F., Self-Organization of Amphiphiles. Langmuir 1988, 4, 3-12.

36. Lee, W. B.; Mezzenga, R.; Fredrickson, G. H., Anomalous Phase Sequences in Lyotropic Liquid Crystals. Physical Review Letters 2007, 99, 187801. 
37. del Mar Graciani, M.; Muñoz, M.; Rodríguez, A.; Moyá, -M,N L., Water Dimethylformamide Alkyltrimethylammonium Bromide Micellar Solutions:Thermodynamic, Structural, and Kinetic Studies. Langmuir 2005, 21, 3303-3310.

38. Moulton, S. E.; Maugey, M.; Poulin, P.; Wallace, G. G., Liquid Crystal Behavior of SingleWalled Carbon Nanotubes Dispersed in Biological Hyaluronic Acid Solutions. J. Am. Chem. Soc. 2007, 129, 9452-9457.

39. Badaire, S.; Zakri, C.; Maugey, M.; Derré, A.; Barisci, J. N.; Wallace, G.; Poulin, P., Liquid Crystals of DNA-Stabilized Carbon Nanotubes. Advanced Materials 2005, 17, 1673-1676.

40. Song, W.; Kinloch, I. A.; Windle, A. H., Nematic Liquid Crystallinity of Multiwall Carbon Nanotubes. Science 2003, 302, 1363.

41. Zhang, S.; Kinloch, I. A.; Windle, A. H., Mesogenicity Drives Fractionation in Lyotropic Aqueous Suspensions of Multiwall Carbon Nanotubes. Nano Letters 2006, 6, 568-572.

42. Davis, V. A.; Parra-Vasquez, A. N. G.; Green, M. J.; Rai, P. K.; Behabtu, N.; Prieto, V.; Booker, R. D.; Schmidt, J.; Kesselman, E.; Zhou, W., et al., True Solutions of Single-Walled Carbon Nanotubes for Assembly into Macroscopic Materials. Nat. Nanotechnol. 2009, 4, 830834.

43. Ericson, L. M.; Fan, H.; Peng, H.; Davis, V. A.; Zhou, W.; Sulpizio, J.; Wang, Y.; Booker, R.; Vavro, J.; Guthy, C., et al., Macroscopic, Neat, Single-Walled Carbon Nanotube Fibers. Science 2004, 305, 1447-1450.

44. Sun, Z.; O’Connor, I.; Bergin, S. D.; Coleman, J. N., Effects of Ambient Conditions on Solvent-Nanotube Dispersions: Exposure to Water and Temperature Variation. J. Phys. Chem. C 2009, 113, 1260-1266.

45. Chiang, I. W.; Brinson, B. E.; Smalley, R. E.; Margrave, J. L.; Hauge, R. H., Purification and Characterization of Single-Wall Carbon Nanotubes. The Journal of Physical Chemistry B 2001, 105, 1157-1161.

46. Hegmann, T.; Qi, H.; Marx, V., Nanoparticles in Liquid Crystals: Synthesis, Self-Assembly, Defect Formation and Potential Applications. Journal of Inorganic and Organometallic Polymers and Materials 2007, 17, 483-508.

47. Lagerwall, J.; Scalia, G.; Haluska, M.; Dettlaff-Weglikowska, U.; Roth, S.; Giesselmann, F., Nanotube Alignment Using Lyotropic Liquid Crystals. Advanced Materials 2007, 19, 359-364.

48. Lagerwall, J. P. F.; Scalia, G., Carbon Nanotubes in Liquid Crystals. Journal of Materials Chemistry 2008, 18, 2890-2898.

49. Lynch, M. D.; Patrick, D. L., Organizing Carbon Nanotubes with Liquid Crystals. Nano Letters 2002, 2, 1197-1201.

50. Zeng, Q.; Cheng, J.; Tang, L.; Liu, X.; Liu, Y.; Li, J.; Jiang, J., Self-Assembled GrapheneEnzyme Hierarchical Nanostructures for Electrochemical Biosensing. Adv. Funct. Mater. 2010, 20, 3366-3372.

51. Sweetman, L. J.; Nghiem, L.; Chironi, I.; Triani, G.; in het Panhuis, M.; Ralph, S. F., Synthesis, Properties and Water Permeability of Swnt Buckypapers. Journal of Materials Chemistry 2012, 22, 13800-13810.

52. Dikin, D. A.; Stankovich, S.; Zimney, E. J.; Piner, R. D.; Dommett, G. H. B.; Evmenenko, G.; Nguyen, S. T.; Ruoff, R. S., Preparation and Characterization of Graphene Oxide Paper. Nature 2007, 448, 457-460.

53. Park, S.; Dikin, D. A.; Nguyen, S. T.; Ruoff, R. S., Graphene Oxide Sheets Chemically Cross-Linked by Polyallylamine. J. Phys. Chem. C 2009, 113, 15801-15804. 
54. Chen, H.; Müller, M. B.; Gilmore, K. J.; Wallace, G. G.; Li, D., Mechanically Strong, Electrically Conductive, and Biocompatible Graphene Paper. Adv. Mater. 2008, 20, 3557-3561.

55. Shin, M. K.; Lee, B.; Kim, S. H.; Lee, J. A.; Spinks, G. M.; Gambhir, S.; Wallace, G. G.; Kozlov, M. E.; Baughman, R. H.; Kim, S. J., Synergistic Toughening of Composite Fibres by Self-Alignment of Reduced Graphene Oxide and Carbon Nanotubes. Nat Commun 2012, 3, 650. 56. Ballard, D. G. H.; Rideal, G. R., Flexible Inorganic Films and Coatings. J Mater Sci 1983, 18, 545-561.

57. Reynolds Iii, R. A.; Greinke, R. A., Influence of Expansion Volume of Intercalated Graphite on Tensile Properties of Flexible Graphite. Carbon 2001, 39, 479-481.

58. Leng, Y.; Gu, J.; Cao, W.; Zhang, T.-Y., Influences of Density and Flake Size on the Mechanical Properties of Flexible Graphite. Carbon 1998, 36, 875-881.

59. Kozlov, M. E.; Capps, R. C.; Sampson, W. M.; Ebron, V. H.; Ferraris, J. P.; Baughman, R. H., Spinning Solid and Hollow Polymer-Free Carbon Nanotube Fibers. Adv. Mater. 2005, 17, 614-617.

60. Steinmetz, J.; Glerup, M.; Paillet, M.; Bernier, P.; Holzinger, M., Production of Pure Nanotube Fibers Using a Modified Wet-Spinning Method. Carbon 2005, 43, 2397-2400.

61. Podsiadlo, P.; Kaushik, A. K.; Arruda, E. M.; Waas, A. M.; Shim, B. S.; Xu, J.; Nandivada, H.; Pumplin, B. G.; Lahann, J.; Ramamoorthy, A., et al., Ultrastrong and Stiff Layered Polymer Nanocomposites. Science 2007, 318, 80-83.

62. Shim, B. S.; Zhu, J.; Jan, E.; Critchley, K.; Kotov, N. A., Transparent Conductors from Layer-by-Layer Assembled Swnt Films: Importance of Mechanical Properties and a New Figure of Merit. ACS Nano 2010, 4, 3725-3734.

63. Olek, M.; Ostrander, J.; Jurga, S.; Möhwald, H.; Kotov, N.; Kempa, K.; Giersig, M., Layerby-Layer Assembled Composites from Multiwall Carbon Nanotubes with Different Morphologies. Nano Letters 2004, 4, 1889-1895.

64. Dalton, A. B.; Collins, S.; Munoz, E.; Razal, J. M.; Ebron, V. H.; Ferraris, J. P.; Coleman, J. N.; Kim, B. G.; Baughman, R. H., Super-Tough Carbon-Nanotube Fibres. Nature 2003, 423, 703-703.

65. Aboutalebi, S. H.; Aminorroaya-Yamini, S.; Nevirkovets, I.; Konstantinov, K.; Liu, H. K., Enhanced Hydrogen Storage in Graphene Oxide-Mwcnts Composite at Room Temperature. Adv. Energy Mater. 2012, 2, 1439-1446.

66. Titelman, G. I.; Gelman, V.; Bron, S.; Khalfin, R. L.; Cohen, Y.; Bianco-Peled, H., Characteristics and Microstructure of Aqueous Colloidal Dispersions of Graphite Oxide. Carbon 2005, 43, 641-649.

67. Aboutalebi, S. H.; Chidembo, A. T.; Salari, M.; Konstantinov, K.; Wexler, D.; Liu, H. K.; Dou, S. X., Comparison of Go, Go/Mwcnts Composite and Mwcnts as Potential Electrode Materials for Supercapacitors. Energ. Environ. Sci. 2011, 4, 1855-1865. 